\title{
Sentimental Perceptualism and the Challenge from Cognitive Bases
}

\author{
Michael Milona (Ryerson University) \\ Hichem Naar (University of Duisburg-Essen)
}

\begin{abstract}
According to a historically popular view, emotions are normative experiences that ground moral knowledge much as perceptual experiences ground empirical knowledge. Given the analogy it draws between emotion and perception, sentimental perceptualism constitutes a promising, naturalist-friendly alternative to classical rationalist accounts of moral knowledge. In this paper, we consider an important but underappreciated objection to the view, namely that in contrast with perception, emotions depend for their occurrence on prior representational states, with the result that emotions cannot give perceptual-like access to normative properties. We argue that underlying this objection are several specific problems, rooted in the different types of mental states to which emotions may respond, that the sentimental perceptualist must tackle for her view to be successful. We argue, moreover, that the problems can be answered by filling out the theory with several independently motivated yet highly controversial commitments, which we carefully catalogue. The plausibility of sentimental perceptualism, as a result, hinges on further claims sentimental perceptualists should not ignore.
\end{abstract}

\section{Introduction}

According to sentimental perceptualism, emotions are normative experiences that ground moral knowledge much as perceptual experiences ground empirical knowledge. This view can be divided into its psychological and epistemological commitments. The psychological commitment is that emotions are experiences of normative properties or relations. The epistemological commitment is that these experiences share with ordinary perceptions the features that make the latter such a good source of knowledge. This paper is about an important class of objections to this epistemological picture. We develop these objections in detail, and maintain that they push sentimental perceptualists toward a series of controversial commitments.

Each of the objections that we consider is rooted in a basic disanalogy between emotions and perceptions, namely that emotions, by contrast with perceptual experiences, always depend for their occurrence on further mental states. For example, I cannot fear the dog in front of me without also perceiving, believing, or remembering that there is a dog in front of me. Philosophers label the mental states to which an emotion responds the 
cognitive base (Deonna and Teroni 2012; Tappolet 2016). We will first be arguing that the mere fact that emotions have cognitive bases is not a problem for sentimental perceptualists. However, the way in which some philosophers - notably Robert Roberts (2013) - have tried to explain why there is no problem do threaten to create trouble. So care is needed. But the most serious difficulties emerge once we delve deeper into the details.

Ralph Wedgwood (2001) and Julien Deonna and Fabrice Teroni (2012) have highlighted several ways in which cognitive bases appear to matter for moral epistemology. According to Wedgwood $(2001,222)$, emotions stand in causal relations to their cognitive base rather than to the normative properties sentimental perceptualists take them to represent. This raises what we call the causal-access problem, according to which emotions lack an epistemologically significant feature of ordinary perception. Another type of concern stems from the fact that the cognitive base of emotions includes not only perceptual states but also beliefs, imaginings, remembrances, etc. In fact, in many cases, emotions respond to representations of contents that are not even in principle perceivable. According to Deonna and Teroni, these observations raise the worry that emotions cannot give us perceptual-like access to their objects if the relation between emotion and value is mediated by states which are themselves not perceptual-like. As we shall see, this worry can take a number of different forms, but they are all instances of what we call the problem of unperceived objects.

In recent years, the causal-access and unperceived objects challenges have been overshadowed by another challenge arising from cognitive bases. Michael Brady (2013) and Deonna and Teroni (2012), among others, argue that cognitive bases not only cause but also justify emotions. But then if emotions are justified or unjustified by their cognitive bases, then emotions are more like judgments than perceptions and thus unsuited to play a foundational epistemological role. But sentimental perceptualists have a variety of natural responses to this objection, including simply denying that emotions are ever, strictly speaking, justified or unjustified (Milona 2016, 903). More complex responses are also available (Cowan 2016; Tappolet 2016).

We argue that the causal-access and unperceived object objections can be answered, but in many cases the commitments sentimental perceptualists need to take on to answer 
the objections are controversial. In the end, then, we hope to arrive at a deeper understanding of the shape that a plausible version of sentimental perceptualism must take. Over the course of the paper, it will become clear that, despite sentimental perceptualism's popularity both historically and today, it is seriously underdeveloped in key details.

\section{Sentimental Perceptualism}

Sentimental perceptualism has a long history. This view, or something very much like it, has been defended by the Aristotle ${ }^{1}$, the Third Earl of Shaftesbury (1991), Francis Hutcheson (1991), Alexius Meinong (1972), and Max Scheler (1973). ${ }^{2}$ Contemporary proponents include Mark Johnston (2001), Graham Oddie (2005), Antti Kauppinen (2013), and Christine Tappolet (2016), among others. The ultimate aim of sentimental perceptualism, as we conceive it, is to demystify moral knowledge: moral knowledge is ultimately rooted in affective experiences just as empirical knowledge is ultimately rooted in perceptual experiences. This epistemological analogy is supported by a second, psychological analogy; affective experiences represent normative properties and/or relations in much the way that perceptual experiences represent properties and relations. Importantly, sentimental perceptualism can fit with a realist picture of value according to which values exist independently of the affective experiences that represent them. For many sentimental perceptualists with realist leanings (e.g., Graham Oddie), a view on which moral experiences are ultimately affective is an attractive alternative to intuitionist moral epistemologies. J.L. Mackie $(1977,38)$ famously objected to appeals to mysterious-sounding "moral intuitions," but perhaps these intuitions are nothing other than familiar forms of affect. ${ }^{3}$ In what follows, we briefly comment on sentimental perceptualism's psychological and epistemological dimensions.

Sentimental perceptualists believe that describing what an affective experience is like (its phenomenology) forces us to refer to normative properties. For example, Sabine

\footnotetext{
${ }^{1}$ For an interpretation of Aristotle along these lines, see Moss (2013).

${ }^{2}$ Shaftesbury's view can be difficult to pin down, but see Gill (2018) for a sentimental perceptualist reading of Shaftesbury.

${ }^{3}$ Audi (2004) and Huemer (2005) argue that appeals to non-affective intuition in moral epistemology are not mysterious. We are officially neutral about the issue here. Our project is internal to the sentimental perceptualist framework.
} 
Döring describes an agent's negative emotional response to a caretaker's harsh punishment of a toddler:

In experiencing indignation at the harsh punishment of the toddler, it seems to you that the punishment is in fact unjust: your occurrent emotional state puts forward your indignation's content as correct. This is in analogy to the content of a sense perception. In perceiving that the cat is on the mat, it seems to you that the cat is actually there. $(2007,377)$

Graham Oddie offers a similar view about desire:

When I desire that P, P has a certain magnetic appeal for me. It presents itself to me as something needing to be pursued, or promoted, or embraced. Now the good just is that which needs to be pursued, or promoted, or embraced. So my desire that P involves P's seeming good (seeming to be worth pursuing). So the desire that $\mathrm{P}$ looks as though it just is the experience of $\mathrm{P}$ as being good. $(2005,41)$

The psychological commitment of sentimental perceptualism is that emotions and/or desires involve non-doxastic experiences of value. ${ }^{4}$ In what follows, we focus primarily on emotions, but the objections and replies that we consider work equally well for sentimental perceptualist theories that emphasize the role of desire.

The epistemological commitment of sentimental perceptualism is that these nondoxastic experiences are the basic source of substantive moral justification and ultimately moral knowledge. Three points about this commitment need clarification. First, the word 'substantive' allows that we may have some conceptual moral knowledge that is not rooted in emotion. For example, it may be a conceptual truth that there cannot be a moral difference without a nonmoral difference. If so, sentimental perceptualists needn't insist that this knowledge is grounded in emotional experience. Conceptual moral knowledge generally isn't considered to be a mystery independent of more general questions about conceptual knowledge.

\footnotetext{
${ }^{4}$ We say 'involve' to avoid adjudicating the question of whether sentimental perceptualists should identify affective experiences with non-doxastic normative representations or whether they should view such experiences as only a part of emotion. This question and its significance are discussed in detail in Milona (ms) and Naar (ms.).
} 
Second, to say that emotions are a basic, or immediate, source of justification means that an emotional experience does not confer justification for believing some normative content because some other state confers justification for believing that content (see Cowan 2015). In this way, emotions function similarly to perceptions, which on many foundationalist approaches are a basic source of justification, and unlike testimony, which is a non-basic source of justification (reliant on some other way of acquiring justification).

Third, we do not assume that sentimental perceptualism is merely a theory about justification. On a phenomenalist view of justification, for instance, if it seems to you that $\mathrm{P}$, then you are defeasibly justified in believing that P. But knowledge seems to require more than this. For example, if due to a brain lesion a person hallucinates a red apple and believes (justifiably) on the basis of that experience that there really is a red apple, then it would not follow that the person knows that there is a red apple. This is because it is an accident that they got it right (Armstrong 1973). Indeed, this gulf between justification and knowledge appears to be why Oddie (2005) defends not only the view that desires represent value but also that they are caused by values. A causal relation, after all, is one of the best candidates for explaining why an experience is non-accidentally correct.

In what follows, we consider the phenomenon of cognitive bases, and whether this presents a problem for the epistemological ambitions of sentimental perceptualism. Our general methodology in developing sentimental perceptualism will be to steer the theory in

the direction which preserves as tight of a connection between moral knowledge and ordinary empirical knowledge as is reasonable, for we take this analogy to be one of the main attractions of the theory.

\section{Cognitive Bases}

Even if emotions share some psychological similarities with ordinary perceptions, they are not perfectly similar. The question is whether any disanalogies between emotion and perception matter for the sentimental perceptualist's epistemological project. Some differences arguably won't. For example, emotional phenomenology is typically valenced pleasurable or painful or a mix - while ordinary perceptual experiences are not. But this difference is not a problem. The valence of emotion may even be important for explaining 
how it is emotions represent normative properties. However, other disanalogies are threatening. For example, some opponents of sentimental perceptualism insist that in describing a perceptual experience, we inevitably describe what we represent - the grass, a dog, etc. - but in emotional experience we do not. In emotional experience, we simply describe internal bodily sensations. If this is right, it may matter for sentimental perceptualists who believe that the presentational character of ordinary perception is significant. Thus there is an ongoing debate (largely a stalemate) about the phenomenology of emotion (see, for example, Dokic and Lemaire 2013; Roberts 2013, 72).

We now turn to an underexplored and potentially significant disanalogy between emotions and perceptions, namely that while emotions are responses to other cognitions, perceptions are not. Deonna and Teroni have done significant work articulating this difference, though the objection is murky in ways we will be trying to get clear about throughout this paper:

Perception constitutes an autonomous way of accessing the properties and objects it represents: seeing, hearing, or touching do not latch onto some prior apprehension of the properties or objects they give access to. In order for a subject to be in a position to see the redness of a tomato, or hear the pitch of a given voice, no prior access to such properties needs to be secured. Emotions work differently, however. They latch onto other psychological states, which, (...) may be of many distinct types (beliefs, memories, imaginative or perceptual experiences) (...). In order for one to be afraid of a dog, one needs some representation of the dog that is logically prior to the emotion itself: one needs to see, hear, or have beliefs about it. (2012, 24-25)

To say that emotions have a cognitive base is not to say that emotions are only responses to cognitions. Our emotions can also be affected by desires, character traits, and moods (Deonna and Teroni 2012, 95-96). In this paper, we consider the extent to which it matters for sentimental perceptualism that emotions are responses to representational mental states. ${ }^{5}$ Deonna and Teroni are explicit that they take this to be a serious problem:

\footnotetext{
5 We are assuming that perceptual experiences are representational, but some philosophers deny this (Campbell 2002). Readers who deny perceptual states represent can translate our arguments accordingly; nothing of what we argue ultimately depends on this assumption.
} 
Let us finally set out what in our eyes constitutes the most serious objection to a perceptual analysis. Even if emotions are to be seen as independent or sui generis ways of accessing the evaluative properties that they reveal, they differ from perceptions in that they cannot be seen as independent ways of accessing the objects that exemplify these properties. (69)

These passages from Deonna and Teroni raise two key questions. First, are they correct that perception is autonomous in a way that emotions are not? And, second, if there is this difference, to what extent does it matter for the sentimental perceptualist's epistemological project?

A couple of points require clarification. The first is to specify more precisely what the cognitive basing relation amounts to. The answer is not singular. At the very least, emotions are caused by their cognitive base. For example, a person's belief that there is a bear charging at them may cause their fear. This belief also appears to be part of the explanation for the emotion's intentionality, i.e., that it is about the bear. It may also be that the cognitive base of an emotion makes the emotion intelligible to the agent experiencing it. Sticking with the same example, the belief about the bear will make the fear it generates intelligible. According to some, that belief also justifies the fear. It is plausibly the case that these different relations can come apart. For example, a belief that serves the intelligible-making role may not be what is really causing the emotion (Echeverri 2017).

Our discussion here takes the causal characterization of cognitive bases as its starting point. ${ }^{6}$ When we say that emotions depend on other mental states, then, we mean, at least, that they are caused by other mental states. In the next section we explore the prospects for denying the disanalogy between emotion and perception. While this is

Furthermore, many philosophers take desires and moods to be representational. Those who are attracted to perceptual theories of the emotions may be especially tempted to adopt such a view (see Oddie 2005, Tappolet 2018). For ease of discussion, however, we set aside these complications. We focus on the cognitive bases that have been emphasized by sentimental perceptualism's opponents. For a discussion of the problem of 'motivational bases' for sentimental perceptualism, and a tentative response on behalf of the perceptualist, see Naar (2016).

${ }^{6}$ It may be a conceptual truth that emotions are mediated by other mental states (Greenspan 1995, 194-6). We are neutral about this. 
tempting, we do not believe that it ultimately works. Sentimental perceptualists should accept the fact that while emotions have a cognitive base, perceptions (at least those of the relevant sort) do not; and they must confront the problems this raises head on.

\section{Denying the Disanalogy: The High-Level Strategy}

Robert Roberts (2013) argues that there is not really a disanalogy. This is a tempting response for sentimental perceptualists to make, since it addresses the challenge (and any more refined challenges that may come from it) at its root. Moreover, it aligns with the core sentimental perceptualist method of illuminating emotion by comparing it to perception.

According to Roberts, while it's true that some perceptions do not depend on other cognitions, others do. Emotions should be understood as analogous to those perceptions which have cognitive bases of their own. Here is a principal example:

Our ability to recognize things we've seen before, and to categorize things we haven't seen before depends on memory, and even very straightforward cases of sense perception are mediated by belief or imagination. Consider the visual perception of a motionless rabbit sitting in surroundings that are visually similar in color and texture to itself. One might stare at the scene for minutes, with 20 / 20 vision and in good lighting, and not see the rabbit. Then, upon being induced to believe a rabbit is there, or upon being told to imagine a rabbit in the setting, one comes to see the rabbit within the very same sensory display. The experience resembles that of "seeing" something new (to oneself) in a gestalt drawing. The rabbit "appears" or "takes shape." (2013, 69).

Roberts has in mind a distinction between sensory perception and conceptual perception $(2013,40)$. Visual sensory experiences include experiences of color and shape, among other contents. But as Roberts' example of the rabbit suggests, how we perceive a visual impression of shapes and colors can vary depending on non-perceptual mental states such as memory, belief, and imagination. Roberts labels perceptions that depend on nonperceptual mental states conceptual, since to have such experiences we must be in possession of relevant concepts (e.g., the concept of a rabbit).

Roberts, then, proposes a view on which emotions are construals of value; and construals are analogous to conceptual perceptions, not sensory ones. Fear, for instance, 
might be thought to involve a construal of a threat which is to-be-avoided (see Roberts 2013, 47). A fearful experience requires one to possess certain concepts, e.g., that of a threat and that of to-be-avoided, but this is no trouble for the analogy since some perceptions require the possession of concepts, too.

We call Roberts's proposal the high-level strategy. In the literature on perception, it is now customary to distinguish between low-level and high-level perception. These categories are typically defined in a way that is internal to the ongoing debate about what contents can be perceptually represented. Low-level visual contents are those contents that are uncontroversially part of visual experience (e.g., color and shape). High-level contents are any other contents (e.g., natural kinds and causal relations). Roberts's notion of a conceptual perception follows a familiar model for how high-level perception is possible. On this picture, high-level contents are made possible when background cognitions (e.g., beliefs and memories) influence one's perceptual experiences. This influence - the existence of which remains controversial - is called cognitive penetration (Siegel 2010, 10).

We caution sentimental perceptualists against the high-level strategy. We do so for several reasons. The first is that it arguably "misses the mark." Even if high-level perceptual experiences depend on other cognitions, they do not always depend on other cognitions when they occur, as is the case with emotions. In the case of rabbit perception, for instance, it is plausible that prior cognitions about rabbits help us to develop a recognitional capacity for perceiving rabbits (Siegel 2010, 99). Once the capacity exists, it can operate autonomously. Thus the high-level model fails to fully alleviate the concern that perceptions are autonomous while emotions are not.

The second concern about the high-level model is that it threatens to undermine one of the main advantages perceptualist models of the emotions have over judgmentalist alternatives. According to judgmentalist theories, emotions consist of normative judgments about the object of the emotion (Solomon 1976; Nussbaum 2001). For example, a judgmentalist might characterize fear that $\mathrm{P}$ as a judgment that $\mathrm{P}$ is dangerous. But judgmentalist theories are now widely rejected, for a variety of reasons. One is that they struggle to make sense of the apparent continuity between human and animal emotion. Both humans and animals appear to be capable of emotions such as fear, anger, jealousy, 
lust, etc. But if emotions consist of normative judgments, then it must be that those who experience an emotion possess the relevant normative concepts to make the judgments. But as Christine Tappolet argues, it is doubtful that animals possess the normative concepts necessary to make such judgments (2016; see also Deigh 1994). While Roberts's view does not say that animals make normative judgments, it does seem to require that animals possess normative concepts; and this is precisely what bothers Tappolet and others about the judgmentalist view. ${ }^{7}$

Finally, and most significantly in this context, the high-level model may undermine the sentimental perceptualist's epistemological ambitions. The reason for this is simple. The sentimental perceptualist says that all substantive normative knowledge is rooted in emotion. But then if emotions are only possible given prior normative cognitions, as an account modeled on high-level perception requires (and as Roberts's own rabbit example illustrates), the sentimental perceptualist faces a dilemma. If the normative mental states that make emotions possible are (sometimes) already justified independently of emotion, or are themselves sources of justification, then the sentimental perceptualist's epistemological thesis is false (cf. Cowan 2015, 187-92; Milona 2018). Or, if the normative mental states are neither justified nor a source of justification, the sentimental perceptualist must explain how the emotions they give rise to could be. And it is difficult to see how such an explanation might go.

\section{$4 \quad$ Denying the Significance of the Disanalogy}

In our view, sentimental perceptualists should maintain that a core set of basic emotions are analogous to low-level sensory perceptions. As we have already seen, sentimental perceptualists maintain that emotions involve non-doxastic experiences of normative properties. What the analogy to low-level sensory perceptions adds is that the content of

\footnotetext{
${ }^{7}$ Roberts is aware this objection. His reply is that animals can possess concepts in the relevant sense. For example, a dog experiencing jealousy distinguishes three relevant parties: herself, the beloved, and the rival. The rival is perceived as the rival insofar as they are perceived to be threatening a cherished relationship with the beloved. For an animal to possess the concepts required for jealousy, they need only make these distinctions perceptually. An animal need not be capable of perception-independent thoughts $(2013,90)$. But this is to shift away from the analogy with high-level perception and towards an analogy with low-level perception. This is a move that we ultimately claim sentimental perceptualists should be pursuing (see below).
} 
these experiences is non-conceptual. ${ }^{8}$ But as with all analogies, there are differences; and one of the differences is that emotions, in contrast to low-level perceptions, always have a cognitive base.

An important qualification should be noted. Sentimental perceptualists can still allow that some (non-basic) emotions depend on the possession of normative concepts. To explain, we first need to remember (see section 1) that sentimental perceptualism permits that we have conceptual normative knowledge that isn't epistemically dependent on emotional experience. For example, the conceptual truth that there cannot be a normative difference without a descriptive difference is presumably not something we learn from emotions. Similarly, many philosophers have proposed conceptual links between different normative concepts. To take just one example, Stephen Finlay (2014) proposes that we can analyze the concept of ought in terms of the concept of good. If there are such conceptual connections, sentimental perceptualists can allow that we gain knowledge of properties not represented by basic emotions by combining normative knowledge rooted directly in emotion with background conceptual normative knowledge (Milona 2016, 906-7). It may also be that we come to have non-basic, or "high-level," emotions that represent these other normative properties in the fashion of high-level perception. ${ }^{9}$ But there is no problem here. The only foundational way to gain knowledge of these other normative properties is, according to the sentimental perceptualist, by combining substantive normative knowledge rooted in basic emotions with an understanding of normative concepts.

But is it a problem for sentimental perceptualist if the core set of basic emotions have cognitive bases while low-level sensory perceptions do not? While there may be a problem, we maintain that it is not an obvious one. Deonna and Teroni emphasize that

\footnotetext{
${ }^{8}$ Readers who do not view low-level perceptions as non-conceptual should feel free to translate our proposal to their favored view of low-level perceptions.

${ }^{9}$ See Prinz $(2007,65-68)$ on basic emotions (candidates for which include fear, anger, and sadness, among other) and non-basic emotions (e.g., indignation, which requires the concept of injustice). For an overview of basic emotions in the scientific literature, see Tracy and Randles (2011). According to Tracy and Randles $(2011,398)$, psychologists say that a basic emotion "should be discrete, have a fixed set of neural and bodily expressed components, and a fixed feeling or motivational component that has been selected for through longstanding interactions with ecologically valid stimuli (e.g., the subjective feeling and motivational component of fear is what it is because this response has historically been most adaptive in coping with typical fear elicitors)."
} 
the presence of a cognitive base means that emotions are not an autonomous way of accessing their objects. But, as they also point out, this does not mean that emotions are not an autonomous way of accessing the normative properties sentimental perceptualists hypothesize that they represent; in fact, their own view of emotion seems to claim something very much in the vicinity (Deonna and Teroni 2014). For example, even though a person cannot fear a bear without thinking that there is a bear, they need not have any thoughts about their reasons to avoid the bear prior to fearing the bear. In effect, the initial sentimental perceptualist response to the presence of cognitive bases should be, "So what?"

The "so what?" response plays an important role in this debate. In particular, it handles a more precise formulation of the cognitive basing challenge that Deonna and Teroni propose:

[Emotions] latch onto other psychological states, which, as we have seen, may be of many distinct types (beliefs, memories, imaginative or perceptual experiences), and which function as their cognitive bases... To emphasize just one lesson among several which may be drawn from the fact that emotions can have a great variety of objects, it is now clear that we should challenge the assimilation of emotions to states susceptible of having only one type of content-a conceptual proposition-like content according to the evaluative judgment theory or a non-conceptual one according to the perceptual theory. An alternative to these must thus be found. (2012, 24-25)

Deonna and Teroni observe that emotions can be responses to states with conceptual and non-conceptual content. But, they seem to think, if emotions can be responses to both kinds of contents, then any theory according to which certain emotions have only one kind of content must be wrong. Sentimental perceptualism is supposed to be such a theory.

But is there really a problem here? We think not. On one picture of the relation between an emotion and its cognitive base, the cognitive base is both a cause and a part of an emotion. On another view, the cognitive base causes the emotion but isn't a part of it. Proponents of both of these views can accept the following:

Mixed Contents: Emotional experiences can include a cognitive base with conceptual content even while the normative representation contributed by the emotional appraisal is non-conceptual. 
There is nothing immediately suspicious about Mixed Contents. Emotions may well be complex states capable of being caused by, or even constituted by, both conceptual and non-conceptual cognitions. So long as the normative dimension of an emotional experience is always non-conceptual (for those emotions understood to be analogous to low-level perceptions) there is no problem.

Sentimental perceptualists shouldn't care that the presence of cognitive bases means that emotions aren't perfectly similar to ordinary sensory perceptions. What they should care about is whether something about cognitive bases matters for their epistemological ambitions. Over the next few sections, we consider arguments that cognitive bases do pose such a challenge.

\section{The Causal-Access Problem}

Ralph Wedgwood (2001) argues that emotions are caused by their cognitive bases rather than the particular normative properties that they are about. ${ }^{10}$ If this is right, then the sense in which perceptions are directly about their objects while emotions are not appears to matter quite a bit, for such causal relations plausibly create an epistemologically significant latch between perceptions and the distal properties that they represent.

Wedgwood's argument is rooted in a series of thoughts about what is required for explaining the occurrence of emotions in particular cases. For example, suppose that a mother arrives early to pick her child up from school. Upon arriving, she observes several of her child's classmates mocking and pushing him on the playground. She then becomes angry. What explains the anger? According to Wedgwood, the explanation has to do with cognitive bases and affective dispositions. First, the anger is a response to the mother's nonevaluative perceptions and beliefs about the treatment of her son. Second, the mother is disposed to experience anger in response to a range of contents that includes those contents represented by her current perceptions and beliefs. A sentimental perceptualist will say that anger represents some normative property or relation (e.g., reasons to retaliate), but we do

\footnotetext{
${ }^{10}$ Wedgwood's specific target is Johnston (2001).
} 
not need to appeal to any such property or relation to explain the occurrence of anger. By contrast, in the case of low-level sensory experiences, we do typically need to appeal to causal relations between perceptual experiences and what the experiences represent, at least in typical cases in which those experiences are accurate.

Wedgwood maintains that the disanalogies with perception run even deeper than may at first appear. Because affective experiences are not caused by particular normative properties, we should not think of them as providing us with direct epistemic access to normative particulars. When all goes well, they put us most directly into contact with general normative truths. Here is how he puts it:

$[\mathrm{A}] \mathrm{n}$ affective state does not have immediate access to any particular evaluative fact. Its access to such facts is mediated by our beliefs or experiences about the particular case, and by a rough correspondence between our affective dispositions and certain general evaluative truths or principles. In short, the evaluative truths to which affective states give us most direct epistemic access are not particular, but general. $(2001,223)$

One may wonder why Wedgwood slides from the thought that because particular values do not cause affective experiences to the conclusion that they are not directly about particular values. But a natural way of understanding the transition is to see Wedgwood as having in mind a picture on which perception gets to have specific contents in part by standing in causal relations to the property instances that they are about; and so if affect fails to stand in such relations, it cannot similarly be about particulars. In this way, Wedgwood's challenge is not just to sentimental perceptualism's epistemology, but also its psychological framework according to which emotions are non-doxastic experiences with normative content.

It is important to be clear that a sentimental perceptualist cannot simply go along with Wedgwood's view that emotions give us knowledge of general truths (even setting aside the additional worry about content determination). As Wedgwood points out, "We can explain how sensory perceptions give us reliable epistemic access to facts about our environment, because a perception is actually caused by the very fact to which it gives us 
access" $(2001,222)$. To give up on a causal relation appears to give up on a perceptual model of moral knowledge. ${ }^{11}$

How, then, should the sentimental perceptualist respond? In our view, the presence of cognitive bases does not prevent emotions from standing in a perception-like, causal relation with the particular normative properties that they are hypothesized to be about. To begin, let's step back for a moment and consider some features of ordinary perception. Imagine an observer looking at the circular opening of a jar from directly above. There is a visual experience as of the jar's having a circular opening. Now imagine the agent looking down at the jar from an angle. There is still a visual experience as of the jar's having a circular opening. However, the proximal stimulations - specifically the light arrays on the observer's retinas - are very different. So how does the observer nonetheless represent the same thing? An attractive explanation of this is that the observer's visual system operates according to certain formation principles. ${ }^{12}$ Formation principles describe dispositions, or formation laws, which ensure that certain types of proximal stimulation lead to the distal environment being represented in the same way. Formation principles thus also help to explain how we can be subject to illusions: proximal stimulations of the right sort will cause the representation of certain environmental properties even when those properties are not present.

Now let's ask the following: on the basis of the above, is it plausible to conclude that the distal properties visual experiences are about are never caused by those properties? Transposing what Wedgwood says about affect, the idea would be that visual representations are explained entirely by proximal stimulation and formation laws/dispositions; there is no need to invoke distal properties. But of course this reasoning is mistaken. We often do need to invoke the distal properties to explain the type of proximal

\footnotetext{
${ }^{11}$ The sentimental perceptualist could concede the point and say that the analogy with perception is only at the level of justification. But this would be to place an important limit on the perceptual analogy, leaving advocates of the view with a difficult question about how emotional experiences of value could be nonaccidentally correct and thus ground moral knowledge. Perhaps some plausible views could be offered, but our methodology in this paper is to preserve the analogy as much as possible, using it to develop answers to the most difficult questions about moral knowledge.

${ }^{12}$ We borrow talk of formation principles (and the formation laws those principles describe) from Burge (2010) and Orlandi (2014).
} 
stimulation (e.g., the type that generates visual representations of circularity) and the visual experiences that such stimulation causes. Return to the observer looking at the circular opening of a jar from directly above. It seems as if the experience depends on the presence of the circularity of the jar; and dependency is good evidence of causation (see Yablo 1992; Oddie 2005). We can reasonably expect the following counterfactual to be true: if the jar had not been circular, the observer would not have experienced it as being circular. To be sure, there are ways the jar could have failed to be circular yet the observer still experiences it as such (e.g., a holographic image of a circular jar); but such possibilities only threaten the counterfactual if the possibilities obtain in nearby worlds. If in the nearest possible world in which the jar is not circular the visual experience of circularity does not occur, then, given a standard understanding of the truth conditions of counterfactuals, the relevant counterfactual comes out true.

These remarks about ordinary perceptual experience help us see why Wedgwood's explanations only exclude emotions from being caused by the particular normative properties sentimental perceptualists argue that they represent by halting the explanation at an arbitrary point. The crucial observation above was this: the proximal stimulation is a cause of the visual experience of circularity insofar as it is a proximal stimulation of a certain type, namely the type that triggers visual representations of circularity. If we type the proximal stimulation too narrowly, then the visual representation will not be dependent on it and thus will be a less plausible hypothesis about the cause of the experience. Now return to the bullying scenario. Wedgwood is correct that the mother's indignation is a response to a certain set of mental states, but we must take care not to characterize those mental states too narrowly. If we characterize them too narrowly, the indignation won't actually depend on that set. Suppose the mother sees the kids shove her son into the mud. The indignation does not depend on the perception, so characterized, because the indignation would have arisen had the perception been of the kids taunting him for being unusually tall. This is the crucial point that Wedgwood's account misses and that leads him to treat normative properties as irrelevant to the explanation. The perceptions and beliefs on which the indignation depends are given by the formation principles (descriptions of formation laws/dispositions) for that type of emotional experience. And it may well be that 
a set of perceptions and beliefs within the scope of the formation laws for that kind of experience would not have arisen had normative reasons (e.g., to help or retaliate) not been present. ${ }^{13}$ In sum, then, sentimental perceptualist should add to their picture the following analogy:

Proximal Analogy: The causal relation between certain emotions, their cognitive base, and relevant values is analogous to the causal relation between certain perceptual experiences, proximal stimulations, and relevant empirical properties.

If this is on the right track, the bare presence of cognitive bases does not prevent emotions from standing in causal relations with the particular normative properties that they represent much as the bare presence of proximal stimulations does not prevent perceptions from standing in causal relations with the particular empirical properties that they represent. Wedgwood's argument, therefore, should be rejected.

\section{The Problem of Unperceived Objects}

Now, even if sentimental perceptualists can avoid Wedgwood's causal-access problem by accepting the Proximal Analogy, they are not completely in the clear with respect to other problems of access raised by the phenomenon of cognitive bases. The best and most straightforward cases for sentimental perceptualism are those in which an emotion is based on a perceptual state representing the emotion's object. This is because the access afforded by the sensory perceptual state is sufficiently robust to be assimilated to the access afforded by proximal stimulations in the perceptual case. ${ }^{14}$ Arguably, it is in part because the relevant access is robust enough in both cases that it is reasonable to maintain that the resulting mental state - the emotion or the sensory perception - can immediately justify the relevant beliefs. If the connection between the cognitive base and the thing it represents is much

\footnotetext{
${ }^{13}$ This response assumes that causation is transitive, or at least that it is in these kinds of cases.

${ }^{14}$ But what of conceptual perceptions? We believe that the account of robustness that we offer below may extend to many conceptual perceptions, and if so, emotions that are responses to such perceptions require no special treatment (see section 6.1). But if some conceptual perceptions happen not to fit our model, then they can be treated in the manner of emotions which are responses to beliefs (see section 7). Either way, the issue does not require separate treatment.
} 
less robust - if, for instance, the cognitive base is a belief acquired via testimony - then it is unclear that the causal relation between an emotion and its object would be of the sort that obtains between sensory perceptions and empirical properties (via proximal stimulations). This is significant, as this forces the sentimental perceptualist to tell a distinct story about emotions based on states that enjoy a much less robust relation with the entities they represent than perception-based emotions. In the following three sections, we consider arguments that certain non-perceptual cognitive bases pose a special challenge for the sentimental perceptualist's epistemological project - a challenge we call the problem of unperceived objects. In particular, we consider cases of rememberings, beliefs, and imaginings.

\subsection{Rememberings}

In this section, we discuss the problem raised by cases of emotion directed at remembered objects. We think that something along the lines of the Proximal Analogy can ultimately be deployed to deal with such cases. However, there will be costs. First, doing this will commit the sentimental perceptualist to a rather controversial view of perception, one which has many detractors. Second, a problem will emerge in the course of showing why memory-based emotions do not constitute an insuperable challenge to the sentimental perceptualist's epistemological project. The problem is that the reason why memory-based emotions might be a source of knowledge might cast doubt on the epistemic credentials of emotions based on belief, a problem we will then tackle in the next section. Let us now turn to the problem emotions based on rememberings raise for the sentimental perceptualist's epistemological project.

We have seen that the mere fact that the connection between emotions and normative properties is indirect does not undermine sentimental perceptualism. But we might worry that, in cases where the cognitive base of an emotion is a memory, the connection between the emotion and the normative property it represents is intuitively too indirect for the emotion to ground knowledge of the property. By contrast with perceptionbased emotion, memory-based emotion seems temporally too far away from the relevant normative property to immediately justify normative judgments about its object. 
To be sure, the sentimental perceptualist can agree that the connection between memory-based emotions and their objects is much more indirect than the connection between perception-based emotions and their objects. But they might insist that the indirectness at issue is not a problem for their view. In response to the claim that memorybased emotions are far removed from their object, the sentimental perceptualist might again reply, "So what?". If the indirectness of perception-based emotions is not a problem, why should more indirectness be seen as any more problematic?

It is instructive to compare this response to a claim, made by some theorists, about the possibility of certain forms of mediated perception such seeing through TV screens and photographs (e.g., Mackie 1976; Walton 1984). Here's John Mackie:

Ordinary language calls perception direct if it is of objects we touch with our hands, see by means of rays of light that come to our eyes, and so on, but indirect if the rays of light are reflected from a mirror or if a television camera and screen are inserted into the visual path, and so on. But what is so important about hands and eyes, as opposed to all the other actual or possible stages in the process? Why should directedness thus construed matter? (Mackie 1976, 46)

As illustrated here, Mackie would agree that ordinary perception is always in some sense mediated, even in simple cases of 'direct' perception of shapes and colors. If indirectness per se does not matter, then it becomes unclear why we should exclude mediums - such as photographs and TV broadcasts - which relate to objects in the world in this very indirect way, from the class of things that enable genuine perception of those objects. We seem to be facing a slippery slope:

No one will deny that we see through eyeglasses, mirrors, and telescopes. How, then, would one justify denying that a security guard sees via a closed circuit television monitor a burglar breaking a window or that fans watch athletic events when they watch live television broadcasts of them? And after going this far, why not speak of watching athletic events via delayed broadcasts (...)? These last examples do introduce a new element: they have us seeing past events. (...) We encounter various other differences also, of course, as we slide down the slope. The question is whether any of them is significant enough to justify digging in our heels and recognizing a basic theoretical distinction, one which we might describe as the difference 
between "seeing" (or "perceiving") things and not doing so. (Walton 1984, 252)

According to Walton, there is no deep theoretical distinction between 'directly' seeing an object in front of our eyes and seeing that object through a photograph or a TV screen. About the alleged indirectness of the connection between photographs/televisions and their object, Walton would therefore reply, "So what?". In looking to Walton, the sentimental perceptualist can treat memory as Walton treats a (delayed) TV broadcast; both would be indirect mediums for perception, but for all that, this isn't a reason to deny that they are perceptual mediums.

One may wonder why Walton thinks that photographs and TV screens can give us access to past events in this way. There must be some difference between a photograph of a scene and (e.g.) an artist's drawing of the scene that would explain why the former, but not the latter, allow genuine perception of the scene. To account for the perceptionallowing aspect of photographs, Walton appeals to the way in which photographs are produced. Minimally, a photograph must have been caused by the scene it represents. This is however not sufficient to distinguish photographs from drawings, which also can be caused by the scene they depict. According to Walton, the feature that makes a photograph a perceptual medium is the fact that it is produced "in a purely mechanical [or automatic] manner" (261), whereas objects cause drawings via a process involving the artist's beliefs about them. The drawing, therefore, functions very much like a piece of testimony, and testimony only supplies justification in some domain if there is some non-testimonial way of acquiring such knowledge. This is very different from the photograph case, as we don't need to think about the photographer's beliefs in any way to be justified in believing the content of the photograph. Upon looking at the photograph, Walton maintains, we are immediately (if defeasibly) justified in believing its content to be true.

Walton thus provides an explanation of why some mediated experiences immediately justify judgment while others don't by claiming that the former must be mediated by what he calls 'transparent' states, where transparency is a matter of satisfying Walton's constraints on perceptual mediation: 
Transparency: A representational state transparently represents an object only when (i) it has been caused by that object and (ii) it has been caused by that object in a way that is mechanical or automatic (where this is supposed to rule out a detour to anyone's beliefs and attitudes).

What does Walton's view imply regarding emotions based on memory? We think that a case can be made for the case that memory is transparent in Walton's sense. First off, although there are debates about the nature of memory, the following claim looks plausible: when an instance of remembering is successful (rather than merely apparent), a causal chain can be traced between the memory and the object of the memory (via the subject's original experience of that object). To be sure, the mere presence of a causal chain is not sufficient (Martin and Deutscher 1966). Suppose that, upon experiencing an event, you describe it to someone and forget about it entirely. A few days later, that person tells you about that event but you forget being told about it. Then you suddenly seem to remember the event, and the reason why you do is because you were told about it. Intuitively, even if there is a causal chain between your apparent memory and the event it represents, it is of the wrong sort; for an instance of remembering to be successful, there must be an appropriate causal connection between the remembering and the remembered object. Notice that, in the case just given, the apparent memory is only apparent because the relevant causal chain involves the testimony of another person and this is in part what disqualifies the apparent memory from being genuine. Arguably, this is because the presence of belief in the causal chain severs the link between the apparent (but veridical) memory and its object in some way. To see this, we can consider a case in which you experience an event but immediately forget about it. A few days later, you for some reason form the belief that the event happened, and then forget that you have formed the belief. Then you suddenly seem to remember the event, but the reason why you do this is because you believe that it happened. Your apparent (but veridical) memory is only apparent is that it lacks the appropriate connection to its object to immediately justify the belief that its content is true.

This contrast between genuine and apparent memories bears a close resemblance to Walton's story about why photographs are transparent while drawings aren't. The 
reason, recall, was that the process by which photographs are produced is 'mechanical' in the sense that it does not involve anyone's beliefs about the world. In this sense, then memory may be claimed to satisfy Walton's mechanical constraint on transparency. If this is right, then memories, when successful, might be just as transparent as photographs, and therefore would immediately justify certain beliefs about their object. If memory is on a par with photographs, TV broadcasts, and other perceptual mediums, then - given that photographs, etc. are for Walton on a par with ordinary, 'direct' perception - there is no obvious reason why we should be suspicious of memory-based emotion.

It is important to note that such a strategy can be seen as an extension of the Proximal Analogy, since an analogy between proximal stimulations and photographs could be used to motivate Walton's mechanical constraint. Indeed, part of what makes the connection between proximal stimulations and the world robust is their isolation from what we happen to believe about the world. Proximal stimulations are 'mechanical' in a similar way photographs are in that they in a sense leave the agent - and the attitudes she happens to hold - out. By employing the slippery slope argument, the sentimental perceptualist can therefore suggest that memory is sufficiently independent of our attitudes to afford the sort of access that both proximal stimulations and photographs may give us. In the end, the sentimental perceptualist need not agree with Walton about photography being a medium for perception (perhaps it is relevant that memory is a natural process and photography is a human invention). But they do need to allow that memory is an indirect medium for perception (or at least that it preserves the epistemic credentials of perception) in much the way that Walton takes photography to be. The alternative would be to deny that emotions in response to memories can be a source of immediate justification, a conclusion which would severely restrict the class of emotions which can be immediate sources of justification and which the sentimental perceptualist is therefore unlikely (rightly, we think) to embrace.

Now, Walton's suggestion as to why photographs constitute a perceptual medium while drawings don't raises a further worry: that, just like our perception of paintings cannot immediately justify any belief about their object, since paintings depend on the aims and beliefs of the painter, emotions based on beliefs cannot immediately justify any further 
beliefs about their object. ${ }^{15}$ This would be a peculiar result, given that our emotions are so often responses to our beliefs about the world. Recall that, for Walton, any belief about an object that a painting of that object might justify will epistemically depend on further beliefs about the individual who made the painting, and the epistemic credentials of their beliefs. The reason is that the process of making the painting involved a person in a way that might have introduced an element of idiosyncrasy, hence of unreliability. An emotion in response to a belief would thereby be analogous to our experience of a drawing. Just as we would need some independent reason to believe that the artist's beliefs about the world are true, we would need some independent reason to believe that the belief that grounds an emotion is true. As a result, any belief we would form in response to the emotion would not be immediately justified. With this problem, we now turn to the case of belief-based emotions.

\subsection{Beliefs}

The foregoing discussion suggests the following constraint on emotions that immediately justify normative judgment:

Transparency Constraint: For an emotion E to immediately justify relevant judgment J, E must be based on a transparent mental state, i.e. a state that is caused by the emotion's object in a purely mechanical or automatic way.

As Walton thinks of it, what it is for a given representational medium to be produced purely mechanically is for it to be produced in a way that is isolated from anyone's rationally evaluable attitudes towards its content. When a representational medium is itself mediated by such attitudes, then for Walton any experience we might have of the medium will not count as genuinely perceptual. To be sure, the question of whether emotions are literally perceptual shouldn't concern sentimental perceptualists. But for Walton, the fact that an experience is grounded in a non-transparent medium implies that it lacks whatever epistemic credentials it has when it is grounded in a transparent medium. The sentimental perceptualist, therefore, might have a reason to worry if her acceptance of Walton's account

\footnotetext{
${ }^{15}$ And one might think that memory is always mediated by belief.
} 
of what we might call 'epistemically acceptable indirectness' commits her to the claim that emotion based on belief never immediately justifies normative judgment.

A first reaction would be to insist that, since beliefs can themselves be justified, it might be sufficient for an emotion to immediately justify a normative judgment that it is based on a justified belief. And although it might initially sound odd to say that an emotion is a perceptual state that can be based on a justified belief, there is nothing obviously wrong with this claim. According to Robert Cowan (2018), emotions that immediately justify normative judgments are sometimes dependent on the presence of justified beliefs as long as such beliefs do not have the same content as the emotion (cf. Siegel 2013). If this is on track, then emotions based on belief do not pose a special epistemological challenge for the sentimental perceptualist, for it is open to her to add the further claim that a belief-based emotion immediately justifies normative judgment only if it is based on a justified belief.

This move, however, comes at potential costs. The first is that it may appear undermotivated: since it is not clear that there are any other cases in which an attitude is an immediate source of justification just in case some other attitude on which it is based is justified, we might wonder why emotions should behave this way. Furthermore, it is no longer clear that the sentimental perceptualist can accept Walton's account of transparency, which we have seen can be of great help to her for developing an account of epistemically acceptable indirectness. It is far from obvious that belief is the sort of thing that can be transparent in Walton's sense. And even if it might sometimes be transparent (see below), the claim at issue here is about nonnormative beliefs in general; it says that, as long as a belief is justified, it does not undermine the epistemic credentials of any emotion it might produce. And many beliefs do not seem to satisfy Walton's conditions on transparency. It appears that beliefs acquired via testimony, even if ultimately caused by their object, involve epistemic agency in a way that makes them distinct from the mechanical processes that produce photographs or TV broadcasts, let alone experiences of the world via eyeglasses and telescopes. If this is right, then a promising way to accommodate the claim that emotions can immediately justify normative judgment even if they are mediated is no longer available to the sentimental perceptualist. 
Since Walton's view provides a good reason for taking the difference between transparent and non-transparent states and mediums seriously, and given that his argument is arguably an extension of the Proximal Analogy we think is important to accommodate perception-based emotions, the sentimental perceptualist is best advised to defend what may seem to be a surprising claim: emotions based on justified beliefs do not (at least typically, see below) immediately justify corresponding normative beliefs. This claim follows from the Transparency Constraint and the plausible claim that belief is not typically transparent. Is this implication problematic? In our view, there does not seem to be anything wrong with granting that a certain class of emotions does not enjoy the relevant epistemic credentials for the reason that their cognitive base does not relate to the world in a mechanical way. Instead of saying that justified beliefs in the cognitive base of an emotion enable the emotion to immediately justify a normative belief, the sentimental perceptualist who accepts the Transparency Constraint will tell a different story: the emotion and belief together form the basis for an inference that the world is normatively as the emotion presents it as being. ${ }^{16}$ Such emotions are in this way unlike low-level perceptions, but they can still play an epistemically useful role, and their lesser status is ultimately not puzzling; it flows in a straightforward way from the nature of their cognitive base.

That being said, there is room for claiming that certain beliefs are transparent in Walton's sense. And indeed such a claim is quite plausible in the case of perceptual beliefs. It is plausible that a belief (e.g.) that there is something red over there, grounded in a certain perceptual experience, might satisfy Walton's constraints on transparency. Most crucially, it is caused by features of the scene in a way that is isolated from further attitudes of the subject. The perceptual belief seems to possess the sort of automaticity that we find in the perceptual experience it is grounded in. ${ }^{17}$ If this is right, then at least some beliefs will be transparent, and therefore any emotion that are based on them would satisfy the

\footnotetext{
${ }^{16}$ Relying for instance on the background belief that if the world is as I believe it to be (non-normatively), then my emotions provide evidence that the world is indeed the way that it presents it as being (normatively). ${ }^{17}$ Walton admits a similar sort of exception with respect to drawings by claiming that " $t$ there are also doodles done automatically, while the doodler's mind is on other things. Some such mechanically executed drawings are probably transparent." (Walton 1984, 267)
} 
Transparency Constraint. But this is not a point on which sentimental perceptualism stands or falls, and we leave it to sentimental perceptualists to decide for themselves which, if any, beliefs count as transparent.

\subsection{Imaginings}

Our emotions are often responses to imaginings. The specific class of imaginings that we focus on are offline analogues of sensory perceptions. Such experiences are distinct from propositional imaginings that are not accompanied by perceptual-like imagery. For example, a person can imagine, in a discursive sense, a situation in which a police officer tells a person that their husband was killed. The connection with emotion in these cases is often thought to be tenuous. By contrast, imagining a police officer delivering this message in a sensory way will tend to trigger emotional responses that are analogous to what the situation would ordinarily trigger. While the emotional response may be weaker, it will still typically be of the same basic sort, e.g., pity (see Van Leeuwen 2017). ${ }^{18}$

The basic worry for sentimental perceptualism is very simple, and targets the epistemological framework of the theory. When we respond emotionally to an imagining, there is no instantiated normative property present. This means that a sentimental perceptualist cannot appeal to the Proximal Analogy to explain the connection between offline emotional experiences and the normative properties that they represent, for there is no such property to function as cause. Furthermore, it is implausible that emotions constitute a window into the world of Platonic essences, even if such essences exist. ${ }^{19}$ But remember that it is a standard assumption in moral philosophy that a priori knowledge is possible. For example, we don't need to see Robin Hood to consider whether he was right to steal from the rich to give to the poor; and, indeed, he needn't ever have existed. It's enough to contemplate his actions. The task for the sentimental perceptualist is to explain

\footnotetext{
${ }^{18}$ Some theorists treat offline emotions as different in kind due to their different functional characteristics (Doggett and Egan 2007). We have no objection to this. Nothing we argue here turns on this debate.

19 Elijah Chudnoff (2013) proposes a theory on which we can intellectually perceive abstracta, including normative properties such as wrongness. But for reasons explained in Milona (2017), we do not believe that this is an attractive model for sentimental perceptualists.
} 
how this could be. But it's not clear how perceptual-like moral emotions can explain the possibility of a priori moral knowledge.

Consider a strategy that sentimental perceptualists might be tempted to pursue, one that is dismissive of the challenge and that we ultimately caution against. A sentimental perceptualist might say that it is only online emotions that are ultimate sources of normative knowledge. On one way of developing this thought, we begin with the apparent truth that imaginary objects do not exist. If they do not, there is nothing there to have justified beliefs about. The non-existence of the object will therefore constitute a defeater for any emotion we might experience in response to an imagining. If this is right, a sentimental perceptualist could model emotions towards imaginary objects on perceptual illusions:

Illusion Analogy: Emotions directed towards imaginary objects are analogous to perceptual illusions.

Armed with this thesis, the sentimental perceptualist may go on to tell a story about how we can use illusory emotions to help us obtain knowledge of normative properties. Such knowledge just won't be basic in the way many philosophers have assumed armchair knowledge could be. We'll have to rely on our foundational (online) emotional experiences to develop strategies for correcting for the illusions.

The trouble with this strategy isn't merely that it leads to an awkward asymmetry between offline and online normative inquiry. It also generates implausible results about fitting emotions. Sentimental perceptualists typically understand a fitting emotion to be an accurate one (see D'Arms and Jacobson 2017 on the pervasiveness of this view among those who think emotions are to be analyzed in terms of representations of values). Paired with the Illusion Analogy, then, this picture of fittingness generates the result that all emotions in response to imaginings are unfitting. But it doesn't seem right to say that your being indignant in response to an imagined act of cruelty would be unfitting, given that the content of the imagining is exactly what would make the situation cruel were it instantiated. Intuitively, emotions in response to imagined objects can be appropriate or fitting. Of course, there is an open question as to why this might be true, but so long as the sentimental 
perceptualist accepts the Illusion Analogy, she must declare all instances of imagined-based emotions as inappropriate given their illusory character. This, the worry goes, is unacceptable.

In what follows, we explain the theses that a sentimental perceptualist can accept to avoid these problems. We think that a maximally desirable version of sentimental perceptualism will, first, treat emotions in response to imaginings as a source of normative knowledge (in many cases) on a par with online emotional experiences, and, second, will generate the result that emotions in response to imaginings can be accurate. This latter commitment is also crucial for preserving the fittingness of offline emotions, given standard sentimental perceptualist accounts of fittingness.

The question of how sentimental perceptualists should explain a priori knowledge is the tip of an iceberg. Not only is a priori knowledge one of the biggest mysteries in epistemology generally, but sentimental perceptualists have had surprisingly little to say on this front to get us started. This is especially so compared to rationalists, who have had quite a bit to say (see Audi 2004; Huemer 2005). In any case, we propose that the best approach for sentimental perceptualists is going to appeal to a specific hypothesis about the evolutionary function of emotions. It is plausible, or at any rate not obviously wrong, that basic emotions (which we first introduced in section 4 above) have evolved to respond to certain value-indicating contents irrespective of the modality of the mental states - with the relevant contents - that cause them. Thus, it is possible to experience emotions not only in response to perceptions, but also in response to beliefs, rememberings, imaginings, hypothesizings, assumings, and any other state of the full spectrum of cognitions. If there is an evolutionary advantage to experiencing emotions in response to value-indicating contents in general, then the fact that some of the cognitive bases of emotion - imaginings, assumings, and the like - represent non-existent objects should not be seen as a mystery. So the sentimental perceptualist may need to add to their account the following claim:

Evolutionary Tracking: Basic emotions have evolved to respond to certain value-indicating contents independently of whether those contents are represented online or offline. 
It is worth emphasizing an additional reason sentimental perceptualists should take Evolutionary Tracking seriously. As we have seen, there is significant pressure for sentimental perceptualists to treat emotions as analogous to low-level perceptions rather than high-level perceptions. High-level perceptions, assuming that there are such experiences, are thought to acquire their content by the influence of other cognitive states penetrating perceptual experience. Low-level experiences, by contrast, are often thought to gain their content in part by standing in functional relations with what they are supposed to be about (Neander 2012). So if sentimental perceptualist denies Evolutionary Tracking, then they are left with a serious puzzle about how it could be that emotions represent normative properties in the fashion of low-level perception. Mark Schroeder $(2008,127)$ calls this the 'fundamental problem' for such views, and Karl Schafer $(2013,268)$ likewise maintains that it may be 'the most serious problem'. ${ }^{20}$ So for this reason, we think that something like Evolutionary Tracking is the best way to explain how sentimental perceptualism can explain a priori knowledge, and is, in any case, better than resorting to the rather costly Illusion Analogy.

But even if we agree that the Evolutionary Tracking thesis is plausible - and mostly what we have done here is to suggest that sentimental perceptualist likely require it to explain how emotions acquire normative content and how emotions can generate a priori normative knowledge - we still need to understand how emotions from the armchair could be accurate. After all, if emotions in response to imaginings involve a presentation that something is the case, then they are going to invariably be inaccurate. This is because, in, say, imagining something which would be cruel, there is not anything which is cruel. In our view, sentimental perceptualist ought to accept the following:

Modal Inheritance: The modal character of an emotion's normative content shifts depending on the type of mental states in its cognitive base.

On this view, an emotional experience that represents some normative property, N, can represent not only that something is $\mathrm{N}$ but also that it would be $\mathrm{N}$. Indeed, something like

\footnotetext{
${ }^{20}$ Schroeder and Schafer refer specifically to sentimental perceptualist views of desire, but their point goes equally well for sentimental perceptualist views of emotions.
} 
Modal Inheritance is needed to explain how remembrances could be accurate. For example, suppose that a person remembers someone uncaringly stomping on their toe, and then experiences anger in response to the imagining. The anger had better represent that something was wrong, if it is to be accurate.

But why believe that Modal Inheritance is true? For sentimental perceptualists, whether emotional normative representations are sensitive to the modality of the cognitive base depends in part on the phenomenology of emotion. As we have seen (section 1), sentimental perceptualists maintain that when we describe an emotional experience, we are forced to describe the normative way in which the world appears to be. In this way, emotions parallel ordinary sensory experiences; describing a perceptual experience as of a green cube, for example, requires us to describe a green cube. A sensory imagining of a green cube requires us to do the same. But the phenomenology is nevertheless not identical. The former, but not the latter, displays a green cube as present. The sentimental perceptualist should say the same about emotions.

The sentimental perceptualist's case here will largely rest on an appeal to the phenomenology of emotion. We think that case for the phenomenological asymmetry between online and offline emotional experience is reasonable, though not conclusive, at least for those who are already on board with the core sentimental perceptualist idea that a proper description of emotional experience requires reference to a normative property. Compare a case in which a person experiences shame upon imagining lying to their spouse about finances with a case in which they experience shame at actually lying to their spouse about finances. The shame experiences will differ in their intensity, but arguably more still. We might notice this by considering the actions that appear intelligible in light of the different episodes. When the shame is a response to an actual happening, the ashamed party may attempt to make amends, or explain their behavior. But not so in the case of the imagining. This is plausibly because the offline shame episode is presenting what would be shameful to do rather that presenting something as being shameful. To be sure, an opponent of sentimental perceptualism here may object that it is the presence of the other mental states (imaginings versus perceptions) that explains why it is intelligible to act on the shame in one case but not the other; shame as such presents something as being the 
case in both instances. However, the sentimental perceptualist position has the advantage of not requiring us to engage in a peculiar correction of inaccurate offline emotions. After all, it is barely intelligible to, say, flee a bear one merely imagines, but it is not clear why this would be so unintelligible if our emotional responses to imaginings presented normative properties as being the case. Thus Modal Inheritance can help us understand why the realm of imagination is not as scary (or wonderful) as it might otherwise be if sentimental perceptualists were to instead accept the Illusion Analogy, treating offline emotions as a realm of inaccurate normative illusions.

\section{Conclusion}

The challenge from cognitive bases is not a simple challenge. It must be dealt with in a piecemeal way depending on the different types of mental states which may comprise a cognitive base. Our aim has been to offer the first sustained investigation of this constellation of puzzles, and correspondingly the first attempt to make sense of sentimental perceptualism in light of them. In sum, we have argued that sentimental perceptualists should accept the following theses:

Mixed Contents: Emotional experiences can include a cognitive base with conceptual content even while the normative representation contributed by the emotional appraisal is non-conceptual.

Proximal Analogy: The causal relation between certain emotions, their cognitive base, and relevant values is analogous to the causal relation between certain perceptual experiences, proximal stimulations, and relevant empirical properties.

Transparency Constraint: For an emotion E to immediately justify relevant judgment J, E must be based on a transparent mental state, i.e. a state that is caused by the emotion's object in a purely mechanical or automatic way.

Evolutionary Tracking: Basic emotions have evolved to respond to certain value-indicating contents independently of whether those contents are represented online or offline.

Modal Inheritance: The modal character of an emotion's normative content shifts depending on the type of mental states in its cognitive base. 
These are bold claims, ones which we hope to see sentimental perceptualist attend to in the future. For if we are right, the plausibility of the theory hinges on them.

\section{Bibliography}

Audi, R. (2004). The Good in the Right: A Theory of Intuition and Intrinsic Value. Princeton University Press.

Armstrong, David. Belief, truth, and knowledge. New York: Cambridge, 1973.

Brady, M.S. (2013). Emotional Insight: The Epistemic Role of Emotional Experience. Oxford University Press.

Burge, T. (2010). Origins of Objectivity. Oxford University Press.

Campbell, J. (2002). Reference and Consciousness. Oxford: Clarendon Press.

Chudnoff, E. (2013). Intuition. Oxford University Press.

Cowan, R. (2015). "Perceptual Intuitionism," Philosophy and Phenomenological Research 90, 1, 164-193.

Cowan, R. (2016). "Epistemic Perceptualism and Neo-Sentimentalist Objections," Canadian Journal of Philosophy 46, 1, 59-81.

Cowan, R. (2018). "Epistemic Sentimentalism and Epistemic Reason-Responsiveness," in Bergqvist, A. \& Cowan, R. (eds.), Evaluative Perception, Oxford University Press.

D’Arms, J. \& Jacobson, P. (2017). "Whither Sentimentalism? On Fear, the Fearsome, and the Dangerous,” in Stueber, K. \& Debes, R. (eds.), Ethical Sentimentalism: New Perspectives, Oxford University Press.

Deigh, J. (1994). “Cognitivism in the Theory of Emotions," Ethics 104, 824-854.

Deonna, J.A. \& Teroni, F. (2012). The Emotions: A Philosophical Introduction. Routledge.

Deonna, J.A. \& Teroni, F. (2014). "In What Sense Are Emotions Evaluations?" in Roeser, S. \& Todd, C. (eds.), Emotion and Value, Oxford University Press.

Doggett, T. and Egan, A. (2007). "Wanting Things You Don't Want: The Case for an Imaginative Analogue of Desire," Philosophers' Imprint 7, 7-17.

Dokic, J. \& Lemaire, S. (2013). “Are Emotions Perceptions of Value," Canadian Journal of Philosophy 43, 2, 227-247.

Döring, S.A. (2007). "Seeing What to Do: Affective Perception and Rational Motivation," Dialectica 61, 3, 363-394.

Echeverri, S. (2017). "Emotional Justification," Philosophy and Phenomenological Research. doi/abs/10.1111/phpr.12453

Finlay, S. (2014), Confusion of Tongues: A Theory of Normative Language. Oxford University Press.

Greenspan, P. (1995). Practical Guilt: Moral Dilemmas, Emotions, and Social Norms. Oxford University Press.

Gill, M. (2018). "Shaftesbury on Life as a Work of Art," British Journal for the History of Philosophy 26, 6, 1110-1131.

Huemer, M. (2005). Ethical Intuitionism. Palgrave Macmillan. 
Hutcheson, Francis. (1991). in D.D. Raphael (ed.), An Inquiry Concerning the Original of Our Ideas of Virtue or Moral Good (selections). in British Moralists 1650-1800: Hobbes-Gay. Indianapolis: Hackett, pp. 261-299.

Johnston, M. (2001). "The Authority of Affect," Philosophy and Phenomenological Research $63,1,181-214$.

Kauppinen, A. (2013). "A Humean Theory of Moral Intuition," Canadian Journal of Philosophy 43, 3, 360-381.

Mackie, J.L. (1976). Problems from Locke. Oxford University Press.

Mackie, J.L. (1977). Ethics: Inventing Right and Wrong. Penguin Books.

Martin, C.B. \& Deutscher, M. (1966). "Remembering," Philosophical Review 75, 2, 161 196.

Meinong, A. (1972). On Emotional Presentation. trans. Marie-Luise Schubert Kalsi. Evanston:

Northwestern University Press.

Milona, M. (2016). "Taking the Perceptual Analogy Seriously," Ethical Theory and Moral Practice 19, 4, 897-915.

Milona, M. (2017) "Intellect versus Affect: Finding Leverage in an Old Debate," Philosophical Studies 174, 9, 2251-2276.

Milona, M. (2018). "On the Epistemological Significance of Value Perception," in Bergqvist, A. \& Cowan, R. (eds.), Evaluative Perception: Aesthetic, Ethical, and Normative, Oxford University Press, pp. 200-218.

Milona, M. (ms.). "The Attitudinalist Challenge to Perceptualism about Emotion."

Moss, J. (2013). Aristotle on the Apparent Good: Perception, Phantasia, Thought, and Desire. Oxford University Press.

Naar, H. (2016). "Le caractère personnel des émotions", Revue Philosophique de la France et de l'Etranger, special issue "Les motivations affectives", ed. J. Deonna, 141, 2, pp. 197-214.

Naar, H. (ms.). "A Humean Challenge for Sentimental Perceptualism"

Neander, K. (2012). "Teleological Theories of Mental Content," The Stanford Encyclopedia of Philosophy (Spring 2012 Edition), Edward N. Zalta (ed.), URL = <http://plato.stanford.edu/archives/spr2012/entries/content-teleological/>.

Nussbaum, M. (2001). Upheavals of Thought: The Intelligence of Emotions. Cambridge University Press.

Oddie, G. (2005). Value, Reality, and Desire. Oxford University Press.

Orlandi, N. (2014). The Innocent Eye: Why Vision Is Not a Cognitive Process. Oxford University Press.

Prinz, J. (2007). The Emotional Construction of Morals. New York: Oxford University Press.

Roberts, R.C. (2013). Emotions in the Moral Life. Cambridge University Press.

Schafer, K. (2013). "Perception and the Rational Force of Desire," Journal of Philosophy 110, 5, 258-281.

Scheler, M. (1973). Formalism in Ethics and Non-Formal Ethics of Values. trans. Manfred Frings and Roger Frunk. Evanston: Northwestern University Press, 1973.

Schroeder, M. (2008). "How Does the Good Appear to Us?" Social Theory and Practice 34, $1,119-130$. 
Shaftesbury, the Third Earl of. Anthony Ashley Cooper. (1991). in D.D. Raphael (ed), An Inquiry Concerning Virtue, or Merit (selections). British Moralists 1650-1800: Hobbes-Gay. ed. Indianapolis: Hackett, pp. 169-188.

Siegel, S. (2010). The Contents of Visual Experience. Oxford University Press.

Siegel, S. (2013). "The Epistemic Impact of the Etiology of Experience," Philosophical Studies $162,3,697-722$.

Solomon, R.C. (1976). The Passions. University of Notre Dame Press.

Tappolet, C. (2016). Emotions, Values, and Agency. Oxford University Press.

Tappolet, C. (2018). "The Metaphysics of Moods," in Naar, H. \& Teroni, F. (eds.), The Ontology of Emotions, Cambridge University Press, pp. 169-186.

Tracy, J. and Randles, D. (2011). "Four Models of Basic Emotions: A Review of Ekman and Cordaro, Izard, Levenson, and Panksepp and Watt." Emotion Review 3, 4, 397 405.

Van Leeuwen, N. "The Imaginative Agent," in Amy Kind and Peter Kung (ed.), Knowledge through Imagination. Oxford University Press.

Walton, K. (1984). "Transparent Pictures," Critical Inquiry, 11, 2, $246-277$.

Wedgwood, R. (2001). "Sensing Values," Philosophy and Phenomenological Research 63, 1, 215-223.

Yablo, S. (1992). "Mental Causation," Philosophical Review 101, 245-280. 